\title{
Time-Frequency Analysis Using Short Time Fourier Transform
}

\author{
Tatsuro Baba*
}

Baba Professional Engineer Office, 1920-2, Usuba, Otawara, Tochigi 324-0035, Japan

\begin{abstract}
A time-frequency analysis, which represents the time change of a signal, is significant in all fields. Wigner distribution, a short time Fourier transform (STFT), a kernel method, and a characteristic function method etc. are known as a time-frequency analyzing method, and those methods have merits and demerits. STFT method has been used as ultrasound blood-flow imaging for a long time, because it is suitable for a non-stationary signal analysis. In this paper, I investigated how to control a time-frequency resolution of STFT, and evaluated the image quality of non-stationary signals using a point-spread function (PSF). The time-frequency resolution of an image corresponds to the aspect ratio of a pixel. Because of the uncertainty principle of time and frequency, to control the aspect ratio is not easy. The PSF is changed by the parameters, such as a frequency-range, a frequency-resolution, a time-range, a window function, a sampling frequency, etc. I propose the control method that keeps the aspect ratio of PSF constant with expansion and contraction of an image.
\end{abstract}

Keywords: Doppler ultrasound system, point spread function, PSF, short time Fourier transform, time-frequency analysis.

\section{INTRODUCTION}

A signal has both a time expression and a frequency expression. In addition, these mixed time-frequency expression exist. For example two-dimensional mapping showing time change of spectra is known. In the timefrequency expression, the uncertainty principle exists between a time-resolution $\Delta t$ and a frequency-resolution $\Delta f$. If $\Delta t$ is made small and the time response is improved, $\Delta f$ becomes large and spectra become ambiguous. Conversely, if $\Delta f$ is made small and the frequency-resolution is improved, $\Delta t$ becomes large and the time response deteriorates. When a horizontal axis is time and a vertical axis is frequency, the two-dimensional distribution of spectra is called a spectrogram. This is also known as a sonogram in the field of acoustics, as a periodgram in the field of signal processing, and as a Doppler spectrum in the field of medical ultrasound. In this paper, the image quality control method of the spectrogram is investigated. Specifically, the methods of controlling the time-resolution, the frequency-resolution, and the aspect ratio of images are proposed.

\section{TIME-FREQUENCY ANALYSES}

About time-frequency imaging there are a spectrogram represented by STFT $[1,2]$ and a scalogram represented by wavelet $[3,4]$. The former is restrained by the uncertainty principle of time-frequency because it divides a signal into short pieces. Since former has the same observation timewidth in all frequency-range, its time-resolution and the frequency-resolution are uniform. The later changes the observation time-width according to frequency. In the case of low frequency, the observation time-width is lengthened

*Address correspondence to this author at the Baba professional engineer office, 1920-2, Usuba, Otawara, Tochigi 324-0035, Japan; Tel/Fax: +81287-29-2360; E-mail: EZD03014@nifty.ne.jp and the frequency-resolution becomes small. Conversely, in the case of high frequency, the time-resolution becomes small and the frequency-resolution becomes large. The timeresolution and frequency-resolution of wavelet transform are not uniform. Further, the inverse transform to the timedomain signal is possible in STFT or wavelet transform. On the other hand, Wigner distribution that is Fourier transform of time-domain signal correlation is also known as a timefrequency analyzing method. And in Wigner distribution, there are a Cohen class based on the spectrogram, and an affine class based on the scalogram. But the inverse transform to the time-domain signal is impossible $[5,6]$.

An example of the difference between spectrogram and scalogram is shown in Fig. (1). Fig. (1a) shows the chirping waveform of a bush warbler. Fig. $(\mathbf{1 b}, \mathbf{c})$ are the STFT image and the wavelet transform image respectively. In the STFT image with uniform frequency-resolution, harmonics are observed clearly. The ultrasonic Doppler blood-flow signal used in Section 4 is non-stationary like chirping of a bush warbler. STFT method has been used in medical Doppler ultrasonic diagnostic equipment for a long time. In the following sections, I concentrated on investigation of the time-frequency analysis based on the STFT method.

\section{SAMPLING FUNCTION AND PSF OF STFT}

\subsection{View of PSF in Time-Frequency Analysis}

The resolution of time-velocity imaging is controlled by parameters, such as the window-width of fast Fourier transform (FFT), a time-shift, and FFT number. If a discrete Fourier transform (DFT) is used instead of FFT, FFT number is combined into the window-width. Fig. (2b) shows the input function of STFT that has a window function $W n$, FFT number $N$ and a time-shift interval $S n$. The vertical axis is amplitude and the horizontal axis is time. The input of the FFT spectrum processing convoluted by a shah-function 


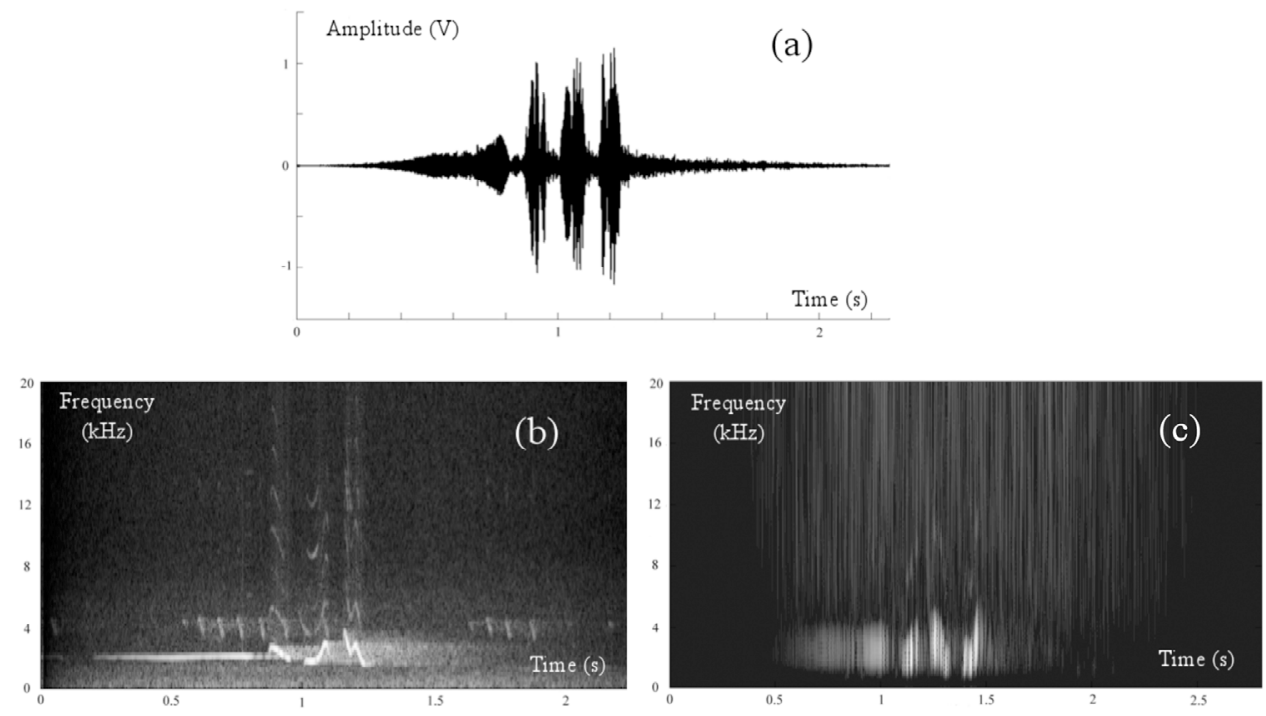

Fig. (1). Signal analyses of a bush warbler chirping. (a) Bush warbler chirping waveform. (b) Spectrogram: Time-frequency imaging by STFT. (c) Scalogram: Time-frequency imaging by wavelet.

(shifting window function) is expressed as $W n(n-k \cdot S n)$. It has the time period $S n$, and $k$ is an integer. When $x(n)$ is an input signal, the time-axis sampling function of STFT can be denoted by equation (1).

$x(n) \otimes W n(n-k \cdot S n)$

Equation (1) is transformed into equation (2) after a Fourier transform is applied.

$X(k, i \omega)=\left(\sum_{n=-\infty}^{+\infty} x(n) \cdot \exp (-j n \omega)\right) \cdot\left(\sum_{n=-\infty}^{+\infty} W n(n-k \cdot S n) \cdot \exp (-j n \omega)\right)$

Here, the first term is an instant spectrum and the second term is equivalent to the transfer function that decides a time-frequency resolution. And $k$ means the $k$-th sampling of STFT (corresponds to the $k$-th spectra output). The energy density of spectra at time $k$ is denoted by equation (3).

$$
P(k, \omega)=|X(k, j \omega)|^{2}
$$

For each different time different spectra can be obtained, and the power of these spectra is the time-frequency distribution of $P(k, \omega)$. The control parameters of timefrequency imaging, $W n$ and $S n$, are shown in Fig. (2b). Fig. (2c) is a two-dimensional sampling function of time and frequency domains, and it corresponds to the response (PSF)

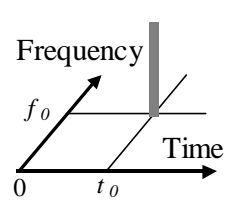

(a)

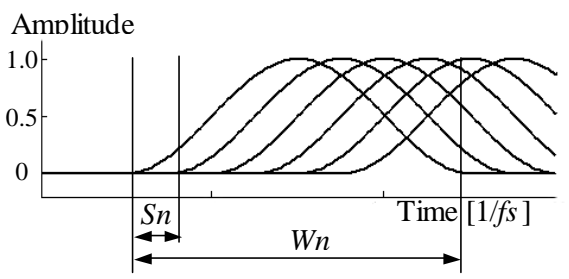

(b) of the short-time impulse input shown in Fig. (2a).

\subsection{Time-Frequency Image Control}

The time-frequency images obtained when changing the parameters of STFT are evaluated in this section. A chirpwaveform suitable for evaluating the time change of spectra is used. A sampling period is set to $1 \mathrm{~ms}$, and sweep-times are set to $5 \mathrm{~s}$ and $0.5 \mathrm{~s}$, and sweep-frequency is set from 20 $\mathrm{Hz}$ to $400 \mathrm{~Hz}$. The chirp-waveform and the STFT images are shown in Fig. (3). Fig. (3b, c) are set 32 and 128 to $N$, respectively. And relative shift-length $S n / N$ is fixed to $1 / 2$. The frequency-resolution becomes small and time-resolution becomes large, when $N$ is large. This follows the uncertainty principle. Fig. (3b, d) are set 32 to $N$, and set $1 / 2$ and $1 / 16$ to $\mathrm{Sn} / \mathrm{N}$ respectively. It turns out that there is no big difference in their image quality and $S n$ hardly influences this case.

\section{IMAGE QUALITY CONTROL}

\subsection{Doppler Ultrasound System}

The examples of image quality control in a Doppler ultrasound system are introduced in this section. In medical ultrasonic diagnostic equipment shown in Fig. (4), there are the tomogram image (B-mode) processing based on reflected echoes, and the spectrum Doppler image processing based on velocity and intensity of blood-flows. Moreover, the parameters shown in Table 1 (except for the adjustment 

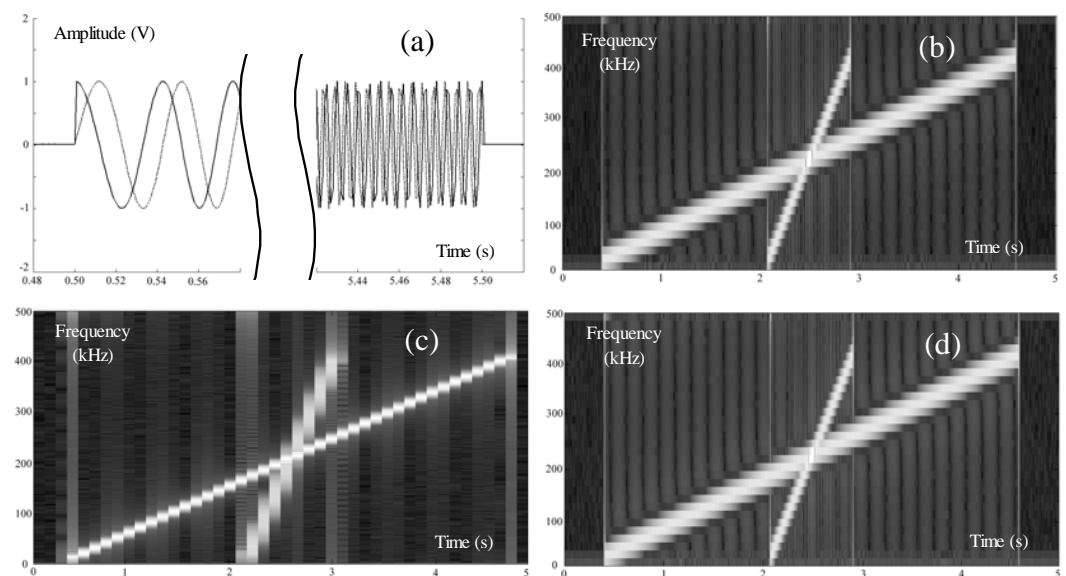

Fig. (3). STFT images of chirp-waveforms. (a) Chirp-waveforms. (b) STFT image ( $N: 32, S n / N: 1 / 2)$. (c) STFT image ( $N: 128, S n / N=1 / 2)$. (d) STFT image $(N: 32, S n / N: 1 / 16)$.

parameters of sensitivity or contrast) are related to image quality of the spectrum Doppler. For example, although users can adjust $f S$ and $S S$, they cannot adjust $D x$ and $D y$ (the parameters fixed by system). If these parameters are changed, the time-frequency resolution changes and image quality of the spectrum Doppler changes. As shown in equation (4), blood-flow velocity $V$ is proportional to Doppler-shift frequency $f d$ [7].

$V=\frac{C}{2 \cdot \cos \theta} \cdot \frac{f d}{f p}$

Here, $f p[\mathrm{~Hz}], C[\mathrm{~cm} / \mathrm{s}]$, and $\theta[\mathrm{deg}]$ are mixer frequency, a sound speed, and an angle between the ultrasound-beam and the blood-flow respectively. These parameters are used to rectify physical quantity of blood-flow velocity.

Fig. (5) shows the example of a Doppler image (the blood-flow of heart) and its PSF. The horizontal axis is time and the vertical axis is velocity corresponding to frequency (Doppler-shift frequency). Fig. (5a) expresses timefrequency distribution of STFT. Contour lines shown in Fig. (5b) has $2 \mathrm{~dB}$ contour interval and its display range (power) is $40 \mathrm{~dB},(-40 \mathrm{~dB}$ to $0 \mathrm{~dB})$. An actual PSF is influenced by power spectrum compression or gradation compensation, so its shape changes a little. In addition, 256 points FFT using Hamming function and zero insertion also affect the PSF.
Medical doctors can choose optimal image quality according to their diagnostic parts or their usages. For example, in abdominal parts, since a blood-flow velocity range is narrow, the granular image that is easy to check the existence of weak blood-flow is desirable. On the other hand, in cardiac parts, since a blood-flow velocity range is large, the image like brushing to the lengthwise direction (fine time-resolution) is desirable.

In this way, spectrum Doppler image quality is affected by the parameters shown in Table 1. When determining image quality, since these parameters are related intricately, their adjustment is difficult. However, I try to control image quality using parameters of STFT this time. I think that image quality can be determined by the sampling function. This PSF changes the time-velocity resolution of the spectrum Doppler image, and can control image quality according to expansion or contraction.

\subsection{Control Modes of Image Quality}

A time expansion/contraction, a frequency expansion/ contraction, and an equal aspect ratio expansion/contraction of time-frequency, are proposed about actual image quality control. Fig. (6) shows the examples of STFT control on these methods. The control algorithm shown in Fig. (6a) has a velocity-resolution preference mode, a time-resolution preference mode, and a time-velocity ratio preference mode.

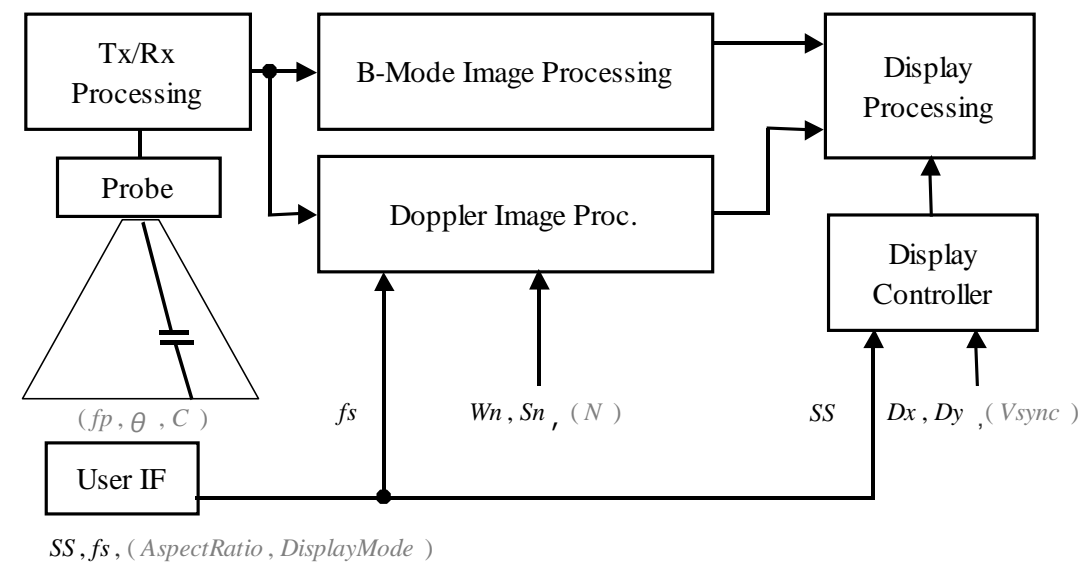

Fig. (4). Medical ultrasonic diagnostic equipment. 
Table 1. Image Quality Parameters

System Parameters that Affect Image Quality

\begin{tabular}{|c|c|c|}
\hline Parameter & Definition & Explanation \\
\hline \hline $\boldsymbol{s} \boldsymbol{S S}$ & Pulse repetition frequency & Tx/Rx pulse wave cycle, and STFT sampling cycle \\
\hline $\boldsymbol{D x}$ & Sweep Speed of time-axis & Scaling or interpolation of STFT output \\
\hline $\boldsymbol{D} \boldsymbol{y}$ & Display width & Number of transverse direction pixels \\
\hline
\end{tabular}

System Parameters that Compensate the Time-Velocity Units

\begin{tabular}{|c|c|c|}
\hline Parameter & Definition & Explanation \\
\hline \hline $\boldsymbol{f} \boldsymbol{\theta}$ & Probe frequency & Quadrature mixer Rx reference frequency \\
\hline $\boldsymbol{\theta}$ & Beam compensation angle & Angle between ultrasound-beam and blood-flow \\
\hline Vsync & Sound speed & Acoustic speed (water) \\
\hline
\end{tabular}

The Control Parameters of Display Aspect Ratio

\begin{tabular}{|c|c|c|}
\hline Parameter & Definition & Explanation \\
\hline \hline $\boldsymbol{S n}$ & STFT shift sampling & STFT sampling period (based on $f s)$ \\
\hline $\boldsymbol{N}$ & FFT number & FFT number $(32,64,128,256$ etc.) \\
\hline $\boldsymbol{W n}$ & Window length & Width of window function for FFT (based on $f s)$ \\
\hline DisplayMode & Display mode & Three kinds of $\Delta T \Delta V \operatorname{control}(* 1)$ \\
\hline AspectRatio & Aspect ratio & Aspect ratio of PSF (in aspect ratio preference mode) \\
\hline
\end{tabular}

$\left({ }^{* 1)}\right.$ Velocity-resolution preference mode, time-resolution preference mode and time-velocity ratio preference mode.

The system parameters $f_{S}, S S$, etc. are considered as input parameters of this control system. The STFT parameters are controlled so that $\Delta T, \Delta V$, or $\Delta V / \Delta T$ become constant. The changes of time-frequency (time-velocity) resolutions by the system parameters are shown in Fig. (6b-d).

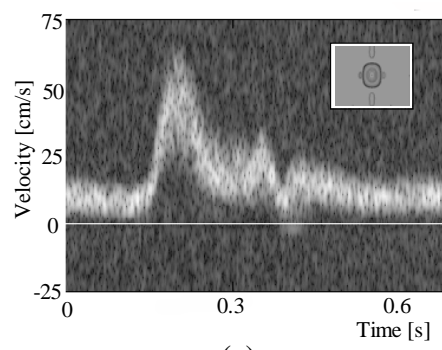

(a)

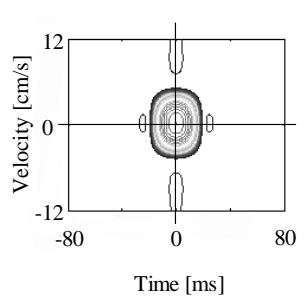

(b)
Fig. (5). Spectrum Doppler image and its PSF. (a) Spectrum Doppler image. (b) PSF: Time-velocity resolution.

A display system is composed of $D x$ [pixel] in time axis, and $D y$ [pixel] in velocity axis. About one pixel dimension, the physical unit of time-direction is $1 /(V s y n c * S S)$ [s] and the physical unit of velocity direction is $2 * \cos \theta^{*} f p^{*} V /\left(N^{*} C\right)$ $[\mathrm{m} / \mathrm{s}]$ (corresponds to the frequency unit of spectrum Doppler output $f s / N[\mathrm{~Hz}])$. The frequency-resolution of PSF $(\Delta V)$ is mainly inversely proportional to $W n$. The time- resolution of PSF $(\Delta T)$ is proportional to $S S$ and $S n$, and inversely proportional to $f_{S}$. This is because the STFT spectrum output cycle is $S n / f_{S}$ [s] and the update cycle of display system is $(V s y n c * S S)$ [s]. STFT spectrum output cycle is hundreds times bigger than the update cycle of display system usually, so the time-axis is smoothed by the moving average etc. The reciprocal of this ensemble length influences the time-resolution of PSF greatly. The details of the control algorithm are described in the reference article [8].

\section{SIMULATIONS}

\subsection{Time-Range Expansion and Contraction}

Carotid blood-flow data was collected using a Pulsed Wave Doppler. After beam-forming processing, Doppler signal extracted by quadrature detection is stored in the IQdata memory. Using the acquired IQ-data, I evaluate the image quality control algorithms. The following conditions are used in simulation. The time-ranges are set to $0.5 \mathrm{~s}, 1.0 \mathrm{~s}$, and $2.0 \mathrm{~s}$ respectively. The carotid blood-flow images generated from IQ-data are shown in Fig. (7). Fig. (7b, e) are the expanded (about 200\%) and contracted (about 50\%) images made by the velocity-resolution preference mode (Fig. 6b). In the case of time-range expansion, as the main purpose is to detect fast blood-flow changing (good time- 

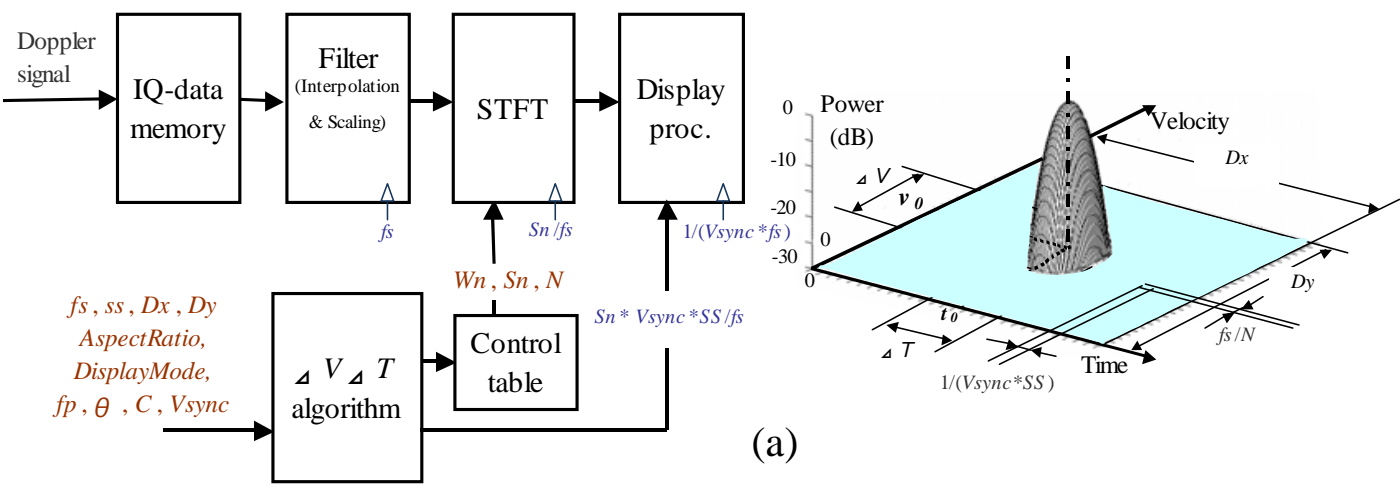

(a)

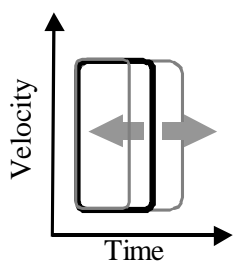

(b)

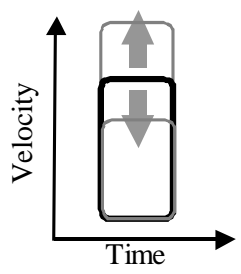

(c)

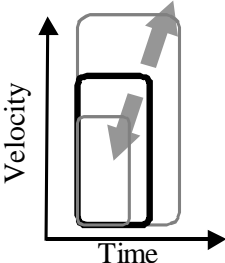

(d)

Fig. (6). Three kinds of image quality control. (a) Input and output of control. (b) Velocity-resolution preference mode. (c) Time-resolution preference mode. (d) Time-velocity ratio preference mode.

resolution), $W n$ is made small. In Fig. (7b) the velocityresolution degrades but the response of velocity-change is good because the time-resolution improves. Conversely, although the time-resolution degrades, the velocityresolution goes up in Fig. (7e) compared with Fig. (7d), and at the same time $\mathrm{S} / \mathrm{N}$ improves. By controlling the parameters of STFT, I can achieve homogeneous images made by the time-velocity ratio preference mode (Fig. 6d).

\subsection{Velocity-Range Expansion and Contraction}

In the previous section, I showed controls of the timerange expansion and contraction of spectrum Doppler
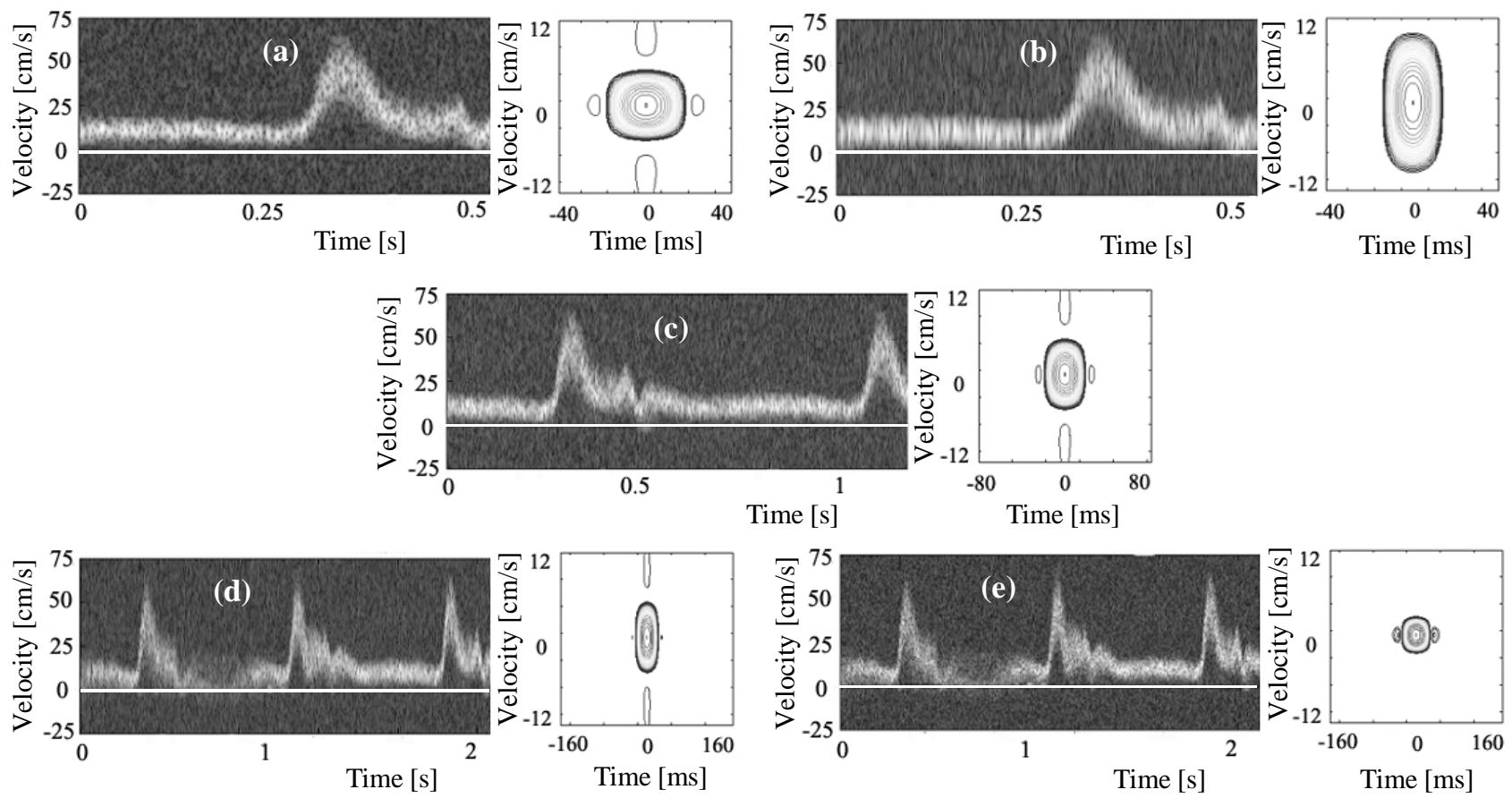

Fig. (7). Time-range expansion and contraction. (a) Expansion of the velocity-resolution preference mode (N: 256, Wn: 48, $S n$ : 4). (b) Expansion of the time-velocity ratio preference mode $(N: 256, W n: 24, S n: 4)$. (c) Original image $(N: 256, W n: 48, S n: 8)$. (d) Contraction of the velocity-resolution preference mode $(N: 256, W n: 48, S n: 12)$. (e) Contraction of the time-velocity ratio preference mode $(N: 256, W n: 72$, Sn: 12). 

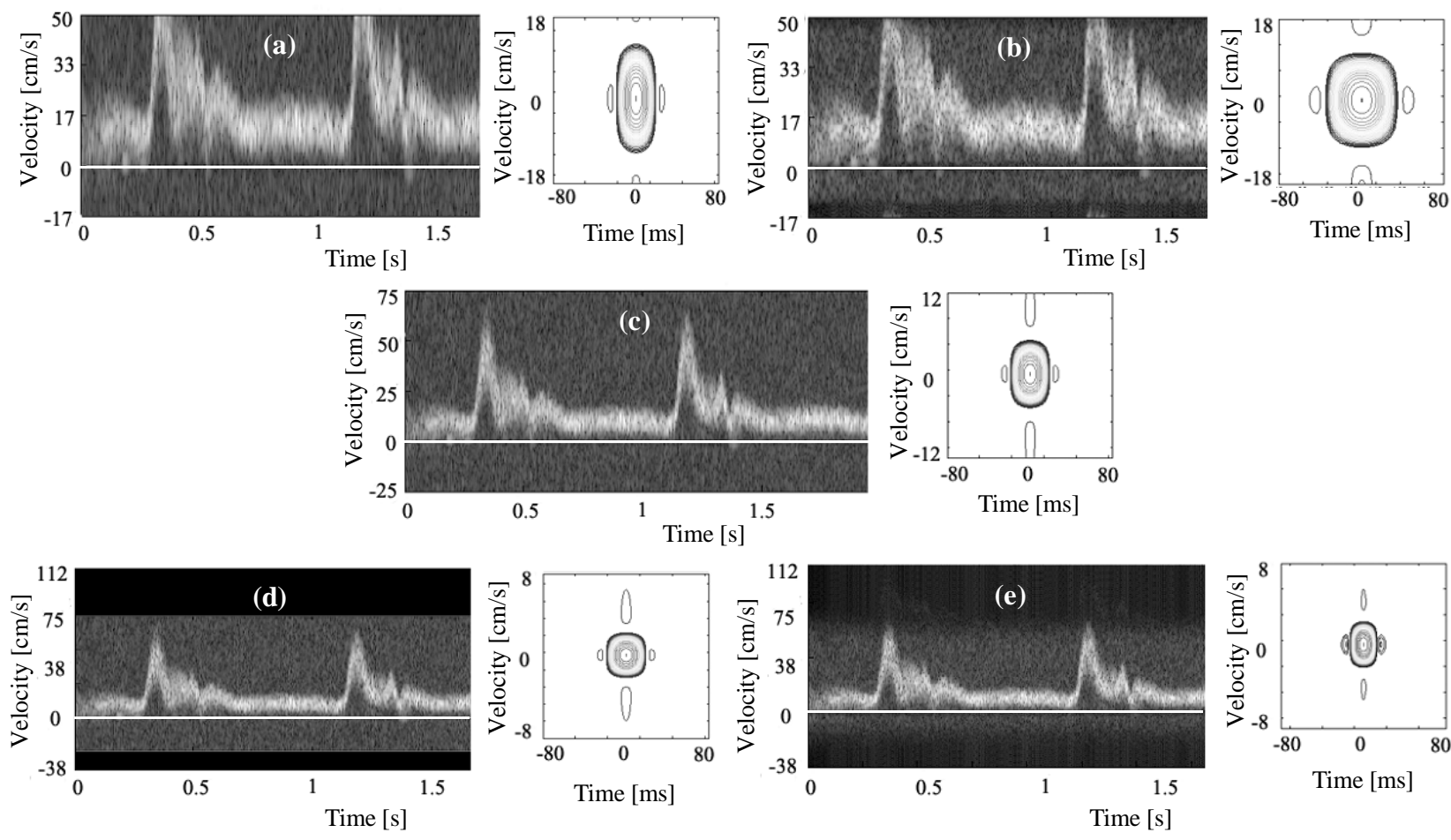

Fig. (8). Frequency-range expansion and contraction. (a) Expansion of the time-resolution preference mode ( $N: 256, W n: 24, S n: 8)$. (b) Expansion of the time-velocity ratio preference mode $(N: 256, W n: 32, S n: 4)$. (c) Original image $(N: 256, W n: 48, S n: 8)$. (d) Contraction of the time-resolution preference mode $(N: 256, W n: 96, S n: 8)$. (e) Contraction of the time-velocity ratio preference mode $(N: 256, W n: 80, S n$ : 12).

images. In this section, I investigate controls of the velocityrange expansion and contraction. Normally, if the expansion or the contraction is processed in acquired data, image quality degrades. I applied the scaling processing of the velocity-range without degradation using IQ-data reconstruction. Fig. (8c) is the original image that was sampled in a velocity range $100 \mathrm{~cm} / \mathrm{s}$. Fig. $(\mathbf{8 a}, \mathbf{d})$ are the expanded $(150 \%)$ and the contracted $(67 \%)$ images made by the time-resolution preference mode (Fig. $\mathbf{6 c}$ ). Fig. $(\mathbf{8 b}, \mathbf{e})$ are the expanded $(150 \%)$ and the contracted $(67 \%)$ images of the time-velocity ratio preference mode (Fig. 6d). In these examples the time-velocity ratio is kept almost constant. I confirmed that an adequate Doppler image could be obtained by the re-processing of STFT.

There are cautions about expansion or contraction of Fig. (8). Since Doppler IQ-signal is once stored in the memory and the frequency-range (corresponds to $f s$ ) is expanded or contracted, a down-sample processing or an up-sample processing are needed. By a scaling and interpolation, generally an aliasing will occur. Since the same signal is needed for comparison, the filtered signal in IQ-data memory is used as the input of STFT in Fig. (8e).

\section{CONCLUSION}

This paper deals with the STFT method to show its suitability for non-stationary analyses, where time-frequency resolution was defined by PSF and a new control method of PSF was also proposed. I developed the reconstruction processing based on acquired IQ-data, and its algorithm optimizes image quality by expanding or contracting of timefrequency axes. As examples, the image quality controls of the ultrasound Doppler blood-flow were evaluated. It became clear that the frequency-resolution is mainly controlled by STFT parameters, and that the time-resolution is mainly controlled by the display parameter like timesmooth. Because there are users's diversions in the setup of image quality, users choose the suitable control mode (a time-resolution preference mode, a velocity-resolution preference mode, and a time-velocity ratio preference mode) according to diagnostic parts. Further, in the time-velocity ratio preference mode, the parameter "aspect ratio" itself is incorporable into a control algorithm of spectrum Doppler imaging. Since the aspect ratio of PSF can be controlled regularly with expansion or contraction, it is natural and comfortable even if compared with expansion or contraction of general images.

\section{ACKNOWLEDGEMENT}

None declared.

\section{CONFLICT OF INTEREST}

The author(s) confirm that this article content has no conflicts of interest.

\section{REFERENCE}

[1] Bracewell RN. The Fourier Transform and its Applications. Boston: McGraw-Hill Companies Inc. 2000.

[2] Semmlow JL. Biosignal and Medical Image Processing. Boca Raton, FL: CRC Press 2009. ISBN-13-978142006230-4. 
[3] Maeda K, Sano A, Takaie H, Hara S. Wavelet Transform and Its Application. Tokyo: Asakura Publishing Co., Ltd. 2001.

[4] Sakakibara S. Wavelet Beginner's Guide. Tokyo: Tokyo Elect. Univ. Publishing Co., Ltd. 1995.

[5] Cohen L. Time-Frequency Analysis New Jersey: Prentice-Hall PTR 1995. ISBN0-13-594532-1.
[6] Sato S, Yoshikawa A, Kiryu T. Introduction to Biosignal Processing. Tokyo: Corona Publishing Co. Ltd. 2004.

[7] Jensen JA. Estimation of blood velocities using ultrasound. NY, USA: Cambridge University Press 1996.

[8] Baba T, Toshiba Corp. Ultrasound Diagnostic Apparatus. Japan Patent 4191812, December 03, 2008.

Received: May 10, 2012

Revised: June 2, 2012

Accepted: June 20, 2012

(C) Tatsuro Baba; Licensee Bentham Open.

This is an open access article licensed under the terms of the Creative Commons Attribution Non-Commercial License (http: //creativecommons.org/licenses/by-nc/3.0/) which permits unrestricted, non-commercial use, distribution and reproduction in any medium, provided the work is properly cited. 